\title{
Réformer avec et pour les jeunes
}

Reforming "with" and "for" young people

Reformar "con" y "para" los jóvenes

\section{Ma-Umba Mabiala et Gisèle Menye Langue}

\section{OpenEdition}

\section{Journals}

Édition électronique

URL : https://journals.openedition.org/ries/9423

DOI : 10.4000/ries.9423

ISSN : 2261-4265

\section{Éditeur}

France Education international

Édition imprimée

Date de publication : 30 avril 2020

Pagination : 177-183

ISBN : 978-2-85420-626-5

ISSN : 1254-4590

\section{Référence électronique}

Ma-Umba Mabiala et Gisèle Menye Lanque, «Réformer avec et pour les jeunes », Revue internationale d'éducation de Sèvres [En ligne], 83 | avril 2020, mis en ligne le 17 juin 2020, consulté le 24 juin 2021. URL : http://journals.openedition.org/ries/9423 ; DOI : https://doi.org/10.4000/ries.9423 


\title{
Réformer avec et pour les jeunes
}

\author{
Ma-Umba Mabiala \\ Expert \\ Gisèle Menye Langue \\ Experte
}

\section{LES JEUNES : UNE PLACE À LA HAUTEUR DE LEUR POIDS DÉMOGRAPHIQUE}

Dans de nombreux pays de l'espace francophone, les jeunes de moins de 35 ans représentent plus de deux tiers de la population. C'est dans les pays d'Afrique subsaharienne que la proportion de jeunes parmi la population totale est la plus importante. En effet, dans les trois quarts des pays de cette région, la proportion de jeunes de moins de 35 ans représente entre 75 et $80 \%$ de la population (OIF, 2018). En République démocratique du Congo (RDC) et à Madagascar, deux pays que nous allons plus spécialement évoquer dans notre analyse, à titre d'exemples, la proportion des jeunes de 15-35 ans représente près du tiers de la population totale du pays (Cedesurk, 2019). L'importance du poids démographique des jeunes dans l'espace francophone rappelle le formidable levier de développement qu'ils peuvent constituer autant que le défi qu'ils peuvent poser. Beaucoup d'efforts ont déjà été consentis par les États, avec l'appui des grands organismes de financement et de coopération multilatérale et bilatérale, tout particulièrement pour améliorer l'accès des jeunes aux services de base jugés prioritaires pour la réduction de la pauvreté, dans le cadre des objectifs de développement durable (ODD), mais aussi en termes d'accompagnement des jeunes (formation, entrepreneuriat, volontariat, numérique, etc.). Toutes ces initiatives ont été prises dans le cadre d'une politique pensée pour les jeunes. Mais, comme l'a montré le rapport d'évaluation des politiques publiques de jeunesse dans les pays francophones (Ouedraogo et al., 2015), les résultats n'ont pas toujours été à la hauteur des espérances, en raison, entre autres, de la non implication des jeunes dans la conception et la mise en œuvre de ces politiques. Les consultations menées par l'OIF dans le cadre de l'élaboration de la Stratégie Jeunesse de la Francophonie le confirment : la faible participation des jeunes aux organes décisionnels est l'un des problèmes majeurs identifiés par les jeunes eux-mêmes. Ces derniers expriment une véritable désillusion vis-à-vis des institutions censées les représenter ou les aider. Il y a une réelle rupture entre les jeunes et les institutions, les premiers ne se sentant pas pris en compte par ces dernières. En France, par exemple, $75 \%$ des jeunes pensent qu'ils ne sont pas reconnus ni entendus par la société ${ }^{1} .76 \%$ des 18-29 ans

1. Selon une enquête menée en avril 2011 par l'Institut CSA pour la Jeunesse ouvrière chrétienne. Source : [https://bit.ly/2QaUYS6] 
disent ne pas faire confiance au gouvernement pour résoudre les problèmes qui se posent à la France. L'analyse longitudinale montre que les écarts entre les jeunes et les plus âgés semblent augmenter depuis 1999, quels que soient les gouvernements ${ }^{2}$. Il est dès lors crucial d'impliquer davantage les jeunes dans la conception, la mise en œuvre et le suivi des dispositifs censés soutenir les réformes. En d'autres termes, il faut « réformer avec et pour les jeunes ». Comment le faire et quel rôle les jeunes peuvent-ils jouer pour mener et pérenniser les réformes éducatives? C'est principalement la réponse à ces deux questions qui constitue la base de notre contribution.

\section{LA FAIBLESSE DES RÉFORMES DANS CERTAINS PAYS}

De notre expérience de responsable d'une direction de l'OIF $^{3}$ et de nos contacts avec les responsables des pays et des ONG avec lesquels nous avons travaillé, nous avons fait le constat suivant. Les administrations avec lesquelles travaillent les organismes de coopération, en particulier dans les pays du Sud, sont constituées de cadres très dévoués mais qui, parfois, font face à d'énormes contraintes (Darbon, 2015). Celles-ci sont de divers ordres. Les ressources font défaut, non seulement des ressources financières mais aussi des ressources humaines qui ne sont pas nécessairement très qualifiées, parce que dans certains pays, ceux qui travaillent dans l'administration publique ne sont pas nécessairement les personnes les plus compétentes. Mais il y a aussi des lacunes liées à la vision même du travail à faire : la vision des réformes à impulser, la vision de la manière dont ces réformes doivent être conçues, élaborées et mises en œuvre. Par conséquent, on observe que, dans la plupart des cas, ce ne sont pas les administrations qui sont les moteurs du changement mais plutôt les acteurs politiques, en particulier les ministres. En effet, dans la plupart des pays du Sud, ces derniers sont détenteurs d'un pouvoir énorme. Comme le note Bisanswa (2019) pour le contexte congolais, un ministre "se perçoit comme un plénipotentiaire, c'està-dire un messie à qui l'entourage doit obéissance, soumission, loyauté et fidélité ». Malheureusement, ces acteurs politiques ont une " durée de vie " très limitée, à cause des contraintes politiques inhérentes aux processus démocratiques dans lesquels ces pays sont engagés.

Nous nous souvenons d'avoir rencontré à Paris, en décembre 2015, un ministre qui, après avoir assisté aux discussions de la COP $21^{4}$ se demandait pourquoi, dans son pays, les jeunes n'apprenaient pas à l'école les notions de base sur le changement climatique et le développement durable. Notre réponse avait été de renvoyer la balle à monsieur le ministre en lui rappelant qu'il avait le pouvoir de décider d'intégrer ces notions dans les curricula pour que, dès le jeune âge ou à l'école secondaire, les enfants puissent apprendre ces notions. C'est ainsi que l'OIF a amorcé un processus d'appui technique au ministère de l'éducation de ce pays pour que de nouveaux contenus soient introduits dans les programmes scolaires, à

2. Source: [http://www.credoc.fr/pdf/Rech/C292.pdf]

3. Nous avons été directeur de l'éducation et de la jeunesse à l’Organisation internationale de la Francophonie, de mai 2013 à fin juin 2019.

4. $21^{\mathrm{e}}$ Conférence des parties de la Convention-cadre des Nations unies sur les changements climatiques (en anglais Conference Of the Parties : COP). La réunion de la COP21 s'est tenue à Paris du 30 novembre au 11 décembre 2015. 
différents niveaux, selon les besoins exprimés par les équipes techniques de ce pays. Malheureusement, environ neuf mois après, il y a eu un remaniement ministériel : le ministre avec lequel nous avions initié ce processus n'était plus en place! Cet exemple illustre le rôle important que jouent les acteurs politiques mais il montre aussi à quel point leur « durée de vie politique » peut être très éphémère.

Vécu par de nombreux responsables, cet exemple pose des problèmes de pérennité, de cohérence systémique et d'efficacité des réformes en faveur des jeunes. En poursuivant notre exemple ci-dessus, nous nous souvenons d'une discussion avec ce ministre qui disait vouloir changer les programmes scolaires pour y inclure des notions sur le changement climatique et le développement durable. Nous lui avions suggéré d'en profiter pour faire une réforme globale, inclure d'autres contenus que son pays souhaitait voir intégrés dans les programmes scolaires parce qu'il n'est pas possible de réviser les programmes chaque année. On ne peut pas non plus changer les manuels scolaires chaque année ; il est par ailleurs nécessaire qu'il y ait cohérence (alignment, diraient les Anglo-saxons) entre les curricula, les supports didactiques et la formation des enseignants. En d'autres termes, quand on s'insère dans une perspective, il faut s'assurer qu'il y ait au moins un certain temps pour penser la réforme, garantir sa mise en œuvre et son efficacité.

Mais que faire si, dans certains pays, les ministres de l'éducation et de la jeunesse ont des «durées de vie » éphémères, si par ailleurs les responsables de l'administration se renouvellent avec chaque nouveau ministre et s'il est souvent de tradition de se démarquer de ce qui a été fait antérieurement ? Faut-il déclarer toute réforme impossible ou se contenter de juxtaposer, au fil des ans, des mini-réformes (des actions ponctuelles) implantées dans un temps très court, le temps de la politique ? Ne vaut-il pas mieux ne pas renoncer à des réformes qui transforment de façon pertinente et durable le système au service des jeunes? Mais comment s'y prendre dans de tels contextes socio-politiques ? C'est à cette dernière question qu'il nous paraît important de répondre.

\section{RÉFORMER PAR LES JEUNES POUR ASSURER LA PÉRENNITÉ}

Face à l'instabilité des responsables politiques et à la faiblesse des administrations, il nous paraît crucial de considérer les jeunes - qui constituent la majorité de la population dans certains pays - comme un levier important pour apporter des changements positifs, et non simplement comme des fauteurs de troubles. Dès lors, comment accompagner les jeunes en tant que levier positif de changement ou, en d'autres termes, comment réformer par les jeunes et pour les jeunes?

Dans les lignes qui suivent, nous décrivons une initiative qui a été pensée et lancée dans le cadre de la programmation quadriennale de l'OIF (2019-2022). Cette initiative consiste à faciliter le dialogue entre les acteurs politiques clefs d'un pays donné, pour qu'ils comprennent les enjeux et défis auxquels sont confrontés les jeunes, les pistes de solutions possibles, les interventions concrètes et pertinentes qui pourraient être menées par les décideurs politiques, à court, moyen et long terme, quels que soient les partis politiques qui sont ou seront au pouvoir. Ces actions doivent être pensées et menées avec les jeunes et pour les jeunes, garants du suivi des actions prévues. 
Les sept étapes clefs de cette initiative peuvent être résumées de la manière suivante.

1) Réalisation d'un état des lieux de la situation des jeunes dans le pays concerné : cet état des lieux vise à dresser un portrait aussi exhaustif et objectif que possible de la situation des jeunes, en partant d'un certain nombre d'indicateurs clefs comme le taux de scolarisation, le taux de diplômés de l'enseignement supérieur, le taux de chômage, l'accès au numérique, etc.

2) Tenue d'un séminaire réunissant les acteurs clefs (toutes tendances confondues), pour discuter de la situation des jeunes et examiner les pistes de solutions possibles, en présence des représentants des jeunes, de la société civile, du secteur privé, du patronat, des partenaires techniques et financiers et du corps diplomatique.

3) Au cours de ce séminaire, les acteurs clefs se mettent d'accord sur un socle commun d'actions et d'interventions concrètes à mettre en œuvre en faveur des jeunes, à court, moyen et long terme.

4) À l'issue de ce séminaire, ces acteurs clefs signent un engagement à intégrer ces actions dans leurs programmes et à les mettre en œuvre ou à contribuer à les mettre en œuvre, qu'ils soient ou non au pouvoir.

5) Après le séminaire, l'OIF et les partenaires techniques et financiers qui le souhaitent s'engagent à contribuer, avec l'aval du gouvernement en place, à mobiliser les ressources pour appuyer la mise en œuvre de ce socle commun d'actions.

6) Les organisations des jeunes sont encouragées à jouer le rôle de veille pour s'assurer que les engagements sont tenus.

7) Au bout d'un certain temps (deux à trois ans), la situation est réévaluée pour examiner les progrès accomplis.

Que penser de cette initiative, suite à l'expérimentation menée dans deux " terrains d'expérimentation ", à Madagascar et en RDC, deux pays où les jeunes constituent une population très importante et où les besoins restent énormes face à l'instabilité des responsables politiques et à la faiblesse de l'administration?

\section{Madagascar et la RDC comme « terrains d'expérimentation »}

Dans les deux pays, conformément aux étapes décrites ci-dessus, le processus a commencé avec la réalisation d'un état des lieux de la situation des jeunes, en partant d'un certain nombre d'indicateurs. Cet état des lieux donnait une photographie objective et exhaustive de la situation des jeunes, y compris dans le secteur de l'éducation et de la formation. D'autres aspects avaient évidemment été pris en compte, notamment les questions de santé sexuelle et de reproduction, à cause du taux relativement élevé des grossesses non désirées chez les jeunes filles, en particulier à Madagascar. Dans les deux pays, cet état des lieux a servi de base de travail au cours d'un séminaire qui a réuni les responsables des partis politiques et les organisations des jeunes, représentées par leurs responsables. À Madagascar, environ 150 jeunes venant de tout le pays et représentant les 22 conseils régionaux de 
la jeunesse avaient pris part à ce séminaire. En RDC, une soixantaine de jeunes issus de la société civile et des partis politiques ont pris part à un séminaire qui a permis d'identifier six axes prioritaires, sur la base de l'état des lieux de la situation des jeunes en RDC, produit par le Centre de documentation de l'enseignement supérieur universitaire et de recherche à Kinshasa (Cedesurk, 2019). Ces axes prioritaires portent sur l'éducation, la santé et la vie familiale, l'emploi et le marché du travail, l'information, la communication et le numérique, la gouvernance et l'engagement citoyen ainsi que le sport, les loisirs et les activités socio-éducatives. En RDC comme à Madagascar, ces séminaires ont permis aux jeunes de proposer des actions pertinentes pour la réalisation des priorités retenues en vue de faire face, à court, moyen et long terme, aux défis et préoccupations auxquels les jeunes sont confrontés, notamment en matière d'éducation et de formation.

À Madagascar, à l'issue des travaux qui se sont déroulés pendant trois jours, du 27 au 29 septembre 2018, le «pacte » a été présenté au président de l'Assemblée nationale et au représentant de l'OIF à Madagascar, qui y ont également apposé leur signature. En RDC, à l'issue de deux jours de travaux, la cérémonie de validation et de signature du « pacte national » a eu lieu le 16 mai 2019, sous la présidence du ministre d'État, ministre de la jeunesse et de l'initiation à la nouvelle citoyenneté, en présence de plusieurs acteurs gouvernementaux, partis politiques et membres de la société civile ainsi que des représentants des partenaires techniques et financiers internationaux.

En termes de contenu, il est significatif de noter la pertinence des propositions contenues dans les pactes nationaux adoptés. Par rapport au secteur de l'éducation, c'est le pacte de la RDC qui contient les propositions les plus intéressantes. À l'instigation des jeunes, ce pacte national a proposé, à titre d'exemples, d'abolir le système de prise en charge des enseignants par les parents d'élèves. En effet, pendant de longues années, face aux dysfonctionnements de l'État, les parents d'élèves ont littéralement pris en charge non seulement le fonctionnement des écoles mais aussi et surtout le financement de tout le système éducatif du pays.

[L]e fonctionnement de toutes les structures et des Bureaux gestionnaires de l'enseignement repose sur des quotes-parts que l'autorité de tutelle leur alloue sur les frais que les parents paient dans les écoles de leurs enfants. (Kalubi, 2019)

Il n'est pas anodin de noter que, plusieurs mois après l'adoption de ce pacte national, le nouveau chef d'État élu a choisi la gratuité de l'enseignement comme réforme la plus importante à mettre en œuvre dans le secteur de l'éducation. D'autres réformes proposées dans le pacte national concernent la mise en place des classes de rattrapage scolaire pour donner une seconde chance aux enfants qui ont abandonné l'école ; la mise en place des mécanismes de dénonciation des violences et harcèlements sexuels dans les milieux scolaires; la redynamisation des classes d'éducation non formelle, afin de répondre aux besoins particuliers des enfants qui vivent dans les zones en situation de conflit.

Dans les deux pays, l'élaboration et l'adoption du pacte national ont été suivies de la mise en place d'un nouveau gouvernement, suite à l'organisation des élections législatives et présidentielles. Certains parlementaires qui, à l'époque, avaient joué un rôle moteur dans ce processus, n’ont pas été réélus. Fort heureusement, quelques-uns l'ont été. Nous espérons donc qu'avec les partis politiques qui 
ont remporté les élections présidentielles et législatives, une dynamique se mettra en place pour tenir les engagements qui ont été pris.

Dans ce processus de dialogue politique, une des étapes clefs est la mise en œuvre du socle d'actions décidées conjointement ; en d'autres termes, la mise en œuvre des réformes. En juin $2019^{5}$, nous n'avions malheureusement pas encore atteint cette étape cruciale. De même, il est prévu qu'à l'étape de mise en œuvre, les jeunes puissent jouer leur rôle de veille, pour vérifier que les " promesses sont tenues ». C'est d'ailleurs l'une des raisons qui avaient motivé les jeunes à prendre part à ce dialogue politique. En effet, certains n’ont pas manqué de relever que les politiciens, en général, promettent beaucoup mais ne réalisent pas ou très peu leurs promesses, une fois au pouvoir. Il était encourageant de voir comment les jeunes se sont engagés à suivre ce processus et à jouer leur rôle de veille pour s'assurer que les engagements qui ont été pris par les acteurs politiques soient respectés et mis en œuvre.

Il faut cependant garder à l'esprit que, aussi bien en RDC qu'à Madagascar, les engagements pris nécessitent des ressources énormes, aussi bien sur le plan technique que sur le plan matériel et financier. C'est un défi majeur qui devrait mobiliser les partenaires techniques et financiers pour accompagner les gouvernements en place en vue de réaliser ces engagements. Nous espérons que cet accompagnement se concrétisera et que des mécanismes de suivi seront mis en place, de sorte qu'il y ait, au bout d'un certain temps - deux, trois ans - une évaluation de la situation pour voir si des progrès ont été réalisés par rapport à la situation de départ.

\section{LA PLACE DES JEUNES DANS LES PROCESSUS DE RÉFORMES}

Bien que cette expérience n'ait pas encore été conduite jusqu'au bout, il est permis d'en tirer quelques enseignements.

L'engagement des jeunes à faire entendre leurs voix dans les domaines qui les concernent est manifeste dans les pays en développement et se traduit par des initiatives porteuses telles que le Pacte national pour la jeunesse ou encore les «Parlements d'enfants ou de jeunes ». Ces cadres d'expression facilitent l'inclusion des jeunes et les amènent à mieux se connaître, à collaborer ensemble pour défendre des droits collectifs et renforcer l'apprentissage d'une citoyenneté active. Cette approche, qui favorise la construction de fondations solides, devrait être promue et soutenue au-delà du milieu urbain, pour asseoir une véritable conscience citoyenne auprès de tous les jeunes.

Les jeunes vivent souvent dans des contextes qui ne sont pas toujours favorables au dialogue politique intergénérationnel, du fait de leur statut de « cadets sociaux ». Par ailleurs, les initiatives qui favorisent le dialogue politique entre les jeunes et les décideurs politiques donnent une visibilité certaine aux acteurs politiques et leur procurent une image d'ouverture vis-à-vis de la jeunesse. Malheureusement, ce dialogue est rarement soutenu par des processus durables, en l'absence d'une veille citoyenne continue de la part des jeunes. En effet, ceux-ci ne disposent ni des moyens techniques ni des moyens financiers nécessaires pour

5. C'est à cette date que nous avons quitté nos fonctions. 
mener les activités de renforcement des capacités et de suivi des plans d'actions qui rendraient leurs interventions efficaces et utiles à la nation ou aux communautés.

Que ce soit au Nord ou Sud, plusieurs États et gouvernements prétendent soutenir les jeunes afin qu'ils puissent prendre part aux décisions dans les domaines qui les concernent. Pourtant, les financements disponibles ne permettent pas de soutenir durablement cette participation au-delà des évènements spéciaux ni de promouvoir une authentique participation citoyenne des jeunes à la vie de la cité. En effet, les États ne considèrent pas souvent que le droit à la participation revêt la même valeur que le droit à l'éducation ou à la santé, d'où des portions congrues dans les budgets des États. Les jeunes étant l'avenir des pays, cet apprentissage réel de la participation citoyenne contribuera sans aucun doute à la stabilité des pays ainsi qu'à la réussite des réformes.

Les exemples suisse et haïtien cités dans le rapport d'évaluation du Programme d'action mondial pour la jeunesse (PAMJ) (Ouedraogo et al., 2015) montrent qu'il ne suffit pas d'encourager l'implication des jeunes, mais qu'il faut aussi les aider à développer leurs capacités en leadership et leurs réseaux afin de garantir l'effectivité de leur participation. Sur ce plan, l'insuffisance, voire l'absence de ressources et l'instabilité financière qui en découlent limitent considérablement les possibilités de participation des organisations des jeunes. Il est donc crucial de veiller à ce que, dans les États du Nord comme du Sud, les jeunes disposent des ressources et outils nécessaires à une participation active aux processus décisionnels concernant les enjeux qui les affectent, en particulier quand il s'agit d'initier et de soutenir des réformes sur le plan local, national et même international.

La participation des jeunes reste donc un enjeu majeur, non seulement par rapport au besoin de pérenniser les réformes mais aussi et, surtout, eu égard à la prise de conscience, par les jeunes, de leurs responsabilités qui se manifeste, de plus en plus, par des mouvements sociaux et politiques pour revendiquer davantage de justice, d'équité et de démocratie, tant dans les pays développés que dans les pays en développement.

\section{BIBLIOGRAPHIE}

BISANSWA J. (2019). « Droit d'entrée et champ universitaire. Pour une cohérence du système universitaire congolais ", Congo-Afrique, $\mathrm{n}^{\circ}$ 537, p. 686-710.

CEDESURK (2019). État des lieux de la situation des jeunes (15-34 ans) en République Démocratique du Congo. Kinshasa.

DARBON D. (2015). « Des administrations africaines paradoxales : entre pratiques locales plurales et régimes d'aide incertains ». Quaderni, $n^{\circ}$ 87, p. 37-50. https://www.cairn.info/ revue-quaderni-2015-2-page-37.htm

KALUBI A. (2019). « La gratuité de l'éducation de base, un défi pour le système éducatif en RD Congo ", Congo-Afrique, $\mathrm{n}^{\circ}$ 537, p. 664-670.

OIF (2018). Rapport sur la situation des jeunes de l'espace francophone, Paris, OIF. https:// jeunesse.francophonie.org/images/OIF_Rapport_jeunesse_2018_web.pdf

OUEDRAOGO A. E. (coord) (2015). Analyse de la mise en ouvre de politiques jeunesse dans les États et gouvernements membres de la Francophonie et exemples de bonnes pratiques. Paris : OIF. [https://bit.ly/2vQHWm7] 